\title{
NONINVASIVE OPTICAL IMAGING OF STAPHYLOCOCCUS AUREUS INFECTION IN VIVO USING AN ANTIMICROBIAL PEPTIDE FRAGMENT BASED NEAR-INFRARED FLUORESCENT PROBES
}

\author{
CUICUI LIU and YUEQING GU* \\ Department of Biomedical Engineering \\ School of Life Science and Technology \\ China Pharmaceutical University \\ Nanjing 210009, P. R. China \\ *guyueqingsubmission@hotmail.com
}

Received 31 May 2013

Accepted 24 June 2013

Published 25 July 2013

\begin{abstract}
The diagnosis of bacterial infections remains a major challenge in medicine. Optical imaging of bacterial infection in living animals is usually conducted with genetic reporters such as lightemitting enzymes or fluorescent proteins. However, there are many circumstances where genetic reporters are not applicable, and there is an urgent need for exogenous synthetic probes that can selectively target bacteria. Optical imaging of bacteria in vivo is much less developed than methods such as radioimaging and MRI. Furthermore near-infrared (NIR) dyes with emission wavelengths in the region of $650-900 \mathrm{~nm}$ can propagate through two or more centimeters of tissue and may enable deeper tissue imaging if sensitive detection techniques are employed. Here we constructed an antimicrobial peptide fragment UBI29-41-based near-infrared fluorescent imaging probe. The probe is composed of UBI29-41 conjugated to a near infrared dye ICG-Der02. UBI29-41 is a cationic antimicrobial peptide that targets the anionic surfaces of bacterial cells. The probe allows detection of Staphylococcus aureus infection $\left(5 \times 10^{7}\right.$ cells $)$ in a mouse local infection model using whole animal near-infrared fluorescence imaging. Furthermore, we demonstrate that the UBI29-41-based imaging probe can selectively accumulate within bacteria. The significantly higher accumulation in bacterial infection suggests that UBI29-41-based imaging probe may be a promising imaging agent to detect bacterial infections.
\end{abstract}

Keywords: Antimicrobial peptide; bacterial targeting; near infrared probe; infection model; in vivo imaging.

This is an Open Access article published by World Scientific Publishing Company. It is distributed under the terms of the Creative Commons Attribution 3.0 (CC-BY) License. Further distribution of this work is permitted, provided the original work is properly cited. 


\section{Introduction}

It has been reported that almost $85 \%$ of critically ill patients in hospitals have fever but show no apparent signs of infection. ${ }^{1}$ Prolonged febrile episodes are often fatal, so the detection of infection at an early stage is important in patient management to achieve a favorable treatment outcome. Staphylococcus aureus ( $S$. aureus) is among the most pathogenic bacteria causing a wide spectrum of diseases ranging from minor wound infections to life-threatening infections such as endocarditis, septicemia, pneumonia and toxic shock syndrome. ${ }^{2}$ Radiological (X-ray computed tomography) and magnetic resonance imaging (MRI) techniques are often not suitable for the early detection of infection because such techniques are focused on specific body parts and provide information only if morphological changes have occurred in the applied areas.

In the case of bacterial targeting, previously reported affinity ligands include antibodies, ${ }^{3}$ sugars, ${ }^{4}$ bacterial binding peptides, ${ }^{5}$ enzyme substrates. ${ }^{6}$ Antibodies are popular, since they can bind tightly to specific molecular targets on the surfaces of both Gram-positive and Gram-negative bacterial cells. While antibodies have the potential advantage of high specificity, their large molecular size $(\sim 150 \mathrm{kD})$ is a limitation for in vivo imaging because of slow tissue diffusion and blood clearance rate. ${ }^{7}$

A more general way to target bacteria is to employ cationic molecules that are electrostatically attracted to the negatively charged cells. ${ }^{9}$ The negative surface charge is a characteristic feature of nearly all bacterial membranes, and results from the high fraction of anionic phospholipids and related amphiphiles. The most striking difference in cytoplasmic membranes between prokaryotic and eukaryotic cells is the composition and topological arrangement of lipids. The surface (outer leaflet) of mammalian cell membranes is exclusively composed of electrically neutral, zwitterionic phospholipids, mainly phosphatidylcholine (PC) and sphingomyelin, whereas bacterial membranes contain large amounts of negatively charged phospholipids, phosphatidylglycerol (PG) and cardiolipin (CL), although their asymmetric distribution between the two leaflets is not well known. The anionic lipids should be exposed to the outer leaflet to a significant extent because the acidic lipid contents sometimes exceed $50 \% .{ }^{8-10}$ The outermembranes of Gram-negative bacteria are covered with polyanionic lipopolysaccharides (LPS).
Another major difference in chemical composition of membranes between prokaryotic and eukaryotic cells is that the latter are abundant in sterols, although some prokaryotes contain sterols or hepanoids, which are similar to sterols. The presence of membranestabilizing cholesterol has been shown to protect human erythrocytes from attack by antimicrobial peptides. $^{8-10}$

UBI29-41 is a cationic human antimicrobial peptide fragment (MW1.69 kDa) with the amino acid sequence Thr-Gly-Arg-Ala-Lys-Arg-ArgMet-Gln-Tyr-Asn-Arg-Arg (TGRAKRRMQYNRR), therefore with 6 positively charged residues (5 Arg + 1 Lys). Recently, technetium-99m $\left({ }^{99 m} \mathrm{Tc}\right)$ labeled UBI29-41 derived from ubiquicidin (UBI) have been widely studied for their capacity to preferentially bind to bacteria without activating leucocytes or mammalian cells. ${ }^{11-17}$ This commonly accepted technique has several advantages, including intrinsically high sensitivity, quantitative capability and clinical translation. However, PET suffers from relatively low spatial resolution, high cost and exposure to ionizing radiation. Therefore, alternatives to radionuclide labeled UBI would be valuable.

Optical imaging of bacteria in vivo is much less developed than methods such as radioimaging and MRI. ${ }^{18}$ One approach is to use bacteria that are genetically encoded to produce luciferase or green fluorescent protein. A second strategy, which is the focus of this study, employs a molecular fluorescent probe. An obvious limitation with a living animal is restricted tissue penetration of the light. However, NIR dyes with emission wavelengths in the region of 650-900 nm can propagate through two or more centimeters of tissue and may enable deeper tissue imaging if sensitive detection techniques are employed. Compared to MRI and radioimaging, optical imaging provides a convenient method to monitor multiple biological processes simultaneously and in real time. ${ }^{19-21}$ Furthermore, the technology is operationally simple, amenable to miniaturization, and potentially mobile. ${ }^{22}$ In this paper, hydrophilic ICG derivative dye ICG-Der-02(excitation/emission 783/ $810 \mathrm{~nm}$ ) with deeper tissue penetration and faster clearance rate $^{23}$ was chosen to conjugate with UBI29-41 peptide to form a NIR probe. We now describe a detailed optical imaging investigation of $S$. aureus infection from the whole animal. We also provide the biodistribution of the probe in vivo. Overall, we find UBI29-41-ICG02 is an effective 
in vivo optical imaging probe for localized infection of $S$. aureus in living mice.

\section{Materials and Methods}

\subsection{Materials}

\subsubsection{Reagent}

UBI29-41 was purchased from China Peptides Co., Ltd. (Shanghai, China). N-hydroxysuccinimide (NHS), dicyclohexylcarbodiimide (DCC) were purchased from Sigma-Aldrich (Shanghai, China). Hydrophilic near-infrared dye ICG-Der-02 (MW995) was prepared in our laboratory. All other reagents used in the study were analytical reagent grade (Shanghai Chemical Reagent Company, Shanghai, China).

\subsubsection{Instruments}

A UV-Visible spectrophotometer (JH 754 PC, Shanghai, China) was used to record the absorption measurements. PHS-25 pH meter (Shanghai, China) was used to measure the $\mathrm{pH}$ values. Sephadex G-15 was used to purify the products. And a high-performance liquid chromatography (HPLC, Waters, USA) system were used to identify the products.

A NIR fluorescence imaging system was used for in vivo real-time imaging of the distribution of the probes in animal subjects. The NIR system is composed of an excitation laser $(\lambda=765.9 \mathrm{~nm}$, NLFC-2.0-763 laser light), a high-sensitivity NIR CCD camera (PIXIS 512B, Princeton Instrumentation) and an $800 \mathrm{~nm}$ long pass filter for capturing the fluorescence from the tissue. In addition, another HLU 32F $400808 \mathrm{~nm}$ laser (LIMO, Dortmund, Germany) was incorporated as background light to obtain the animal profile. Otherwise, the imaging was completely dark except for the fluorescence spot. For image analysis, we used winview software that complied with the NIR imaging system and pseudo color mode was chosen.

\subsection{Methods}

\subsubsection{Synthesis and characterization of UBI29-41-ICG02 conjugates}

ICG-Der-02 ( $5 \mathrm{mg}, 0.005 \mathrm{mmol})$ was reacted with EDC.HCl (2 mg, $0.01 \mathrm{mmol})$ and NHS $(1.73 \mathrm{mg}$,
$0.009 \mathrm{mmol})$ in DMF $(200 \mu \mathrm{L}) .{ }^{23}$ After stirring in the dark for $5 \mathrm{~h}$ at room temperature, the solution was mixed with UBI29-41 dissolved in phosphate buffer solution ( $\mathrm{PBS}, \mathrm{pH}=7.4)$. The mixture was stirred at room temperature overnight in the dark. The crude product UBI29-41-ICG02 was first confirmed by thin layer chromatography (TLC) and subsequently purified by filtration over Sephadex G-15. Finally, the absorbance spectra of UBI29-41-ICG02 was recorded on a UV-Visible spectrophotometer and an NIR spectral system. The chemical purity of the product was determined by analytical HPLC. HPLC solvents consist of water containing $0.1 \%$ tri fluoroacetic acid (TFA; solvent A) and acetonitrile containing $0.1 \%$ TFA (solvent B). The elution protocol started from $65 \% \mathrm{~A}$ and $35 \% \mathrm{~B}$ to $52 \% \mathrm{~A}$ and $48 \% \mathrm{~B}$ over $13 \mathrm{~min}$, held at $52 \% \mathrm{~A}$ and $48 \% \mathrm{~B}$ for $5 \mathrm{~min}$, continued to $42 \% \mathrm{~A}$ and $58 \% \mathrm{~B}$ over $20 \mathrm{~min}$.

\subsubsection{In vitro antibacterial ability of UBI29-41}

To explore whether the antimicrobial peptide fragment UBI29-41 has the antibacterial ability against $S$. aureus, we use the microplate broth dilution method. ${ }^{24}$ Briefly, $S$. aureus from overnight cultures was inoculated into $5 \mathrm{~mL}$ of trypticase soy broth and allowed to enter logarithmic phase (OD 600 $\sim 0.3$ ) after approximately $2 \mathrm{~h}$ of incubation. UBI 29-41 was serially diluted into a final volume of $100 \mu \mathrm{L}$. S. aureus were diluted to a theoretical OD 600 of 0.001 in Mueller Hinton Medium and seeded onto the microplates to produce a final volume of $200 \mu \mathrm{L}$ per well. The OD 600 was measured immediately before placing into an incubated shaker at $37^{\circ} \mathrm{C}$, and then again $\sim 18 \mathrm{~h}$ later. All samples were assayed in triplicate and the survival rate was calculated as follows:

Survival rate $=$ (mean absorbance of test wells mean absorbance of medium control wells)/(mean absorbance of untreated wells - mean absorbance of medium control wells) $\times 100 \%$.

\subsubsection{In vitro cytotoxicity studies of UBI29-41}

To evaluate the biocompatibility of UBI29-41 as imaging ligand, the cytotoxicity of UBI29-41 was investigated by using the MTT assay. ${ }^{25}$ Briefly, human hepatic L02 cells were seeded in a 96-well plate at a density of $5 \times 10^{3}$ cells/well and then 
incubated overnight $2.5 \% \mathrm{CO}_{2}, 37^{\circ} \mathrm{C}$ in a cell culture incubator. After that, the UBI29-41 was added for incubation $24 \mathrm{~h}$ and then the MTT dye $(20 \mu \mathrm{L}$ of $5 \mathrm{mg} / \mathrm{mL}$ ) was added to each well. After incubation for another $3 \mathrm{~h}$ at $37^{\circ} \mathrm{C}$, the MTT working solution was removed. Then, $200 \mu \mathrm{L}$ DMSO was added into each well and the plate was shaken for $20 \mathrm{~min}$ at room temperature. All samples were assayed at $570 \mathrm{~nm}$ in triplicate and the survival rate was calculated as follows:

Survival rate $=($ mean absorbance of test wells mean absorbance of medium control well)/(mean absorbance of untreated wells - mean absorbance of medium control well) $\times 100 \%$.

\subsubsection{Dynamics and biodistribution of ICG-Der-02 and UBI29-41-ICG02 in healthy mice}

BALB/C mice (aged 4-6 weeks and weighed at 18-22 g) were purchased from Qinglongshan animal breeding grounds (Nanjing, China). All animal experiments were carried out in compliance with the Animal Management Rules of the Ministry of Health of the People's Republic of China (document No. 55, 2001) and the Guidelines for the Care and Use of Laboratory Animals of China Pharmaceutical University.

Their abdomen were shaved and depilated. ICGDer-02 and $10 \mathrm{nmol}$ of UBI29-41-ICG02 in $200 \mu \mathrm{L}$ of PBS were intravenously injected normal mice via the tail vein. A noninvasive in vivo continuous fluorescence imaging apparatus was used to assess the distribution of the dyes or probes in mice as described previously. All NIR fluorescent images were respectively acquired from the abdomen and displayed with the same fluorescence axillary fossa of each mouse.

\subsubsection{Dynamics and biodistribution of ICG-Der-02 and UBI29-41-ICG02 in infected mice}

To investigate the targeting ability of the probes, an infection model was established. ${ }^{26}$ Briefly, saline solution $(0.1 \mathrm{~mL})$ containing $10^{7}$ colony-forming units of viable $S$. aureus 25923 were subcutaneously injected into the axillary fossa of each mouse. Twenty-four hours after the inoculation of $S$. aureus, when significant swelling was visible at the injection site, UBI29-41-ICG02 $(0.2 \mathrm{~mL})$ was injected into the tail vein. The mice were imaged with the NIR imaging system at predetermined time intervals within $24 \mathrm{~h}$ postinjection.

\subsubsection{Statistical analysis}

Data are expressed as means standard deviation. Statistical analysis was performed using Student's $t$-test with statistical significance assigned for a $p$-value of $<0.05$.

\section{Results}

\subsection{Synthesis and characterization of UBI29-41-ICG02 probes}

UBI29-41-ICG02 was synthesized by following the procedures described in the Methods Section, as shown in Fig. 1. The purified UBI29-41-ICG02 was evaluated by UV absorption spectrum analysis in PBS with $\mathrm{pH} 7.4$ as shown in Fig. 2(a). The absorption spectra of UBI29-41-ICG02 displayed all of

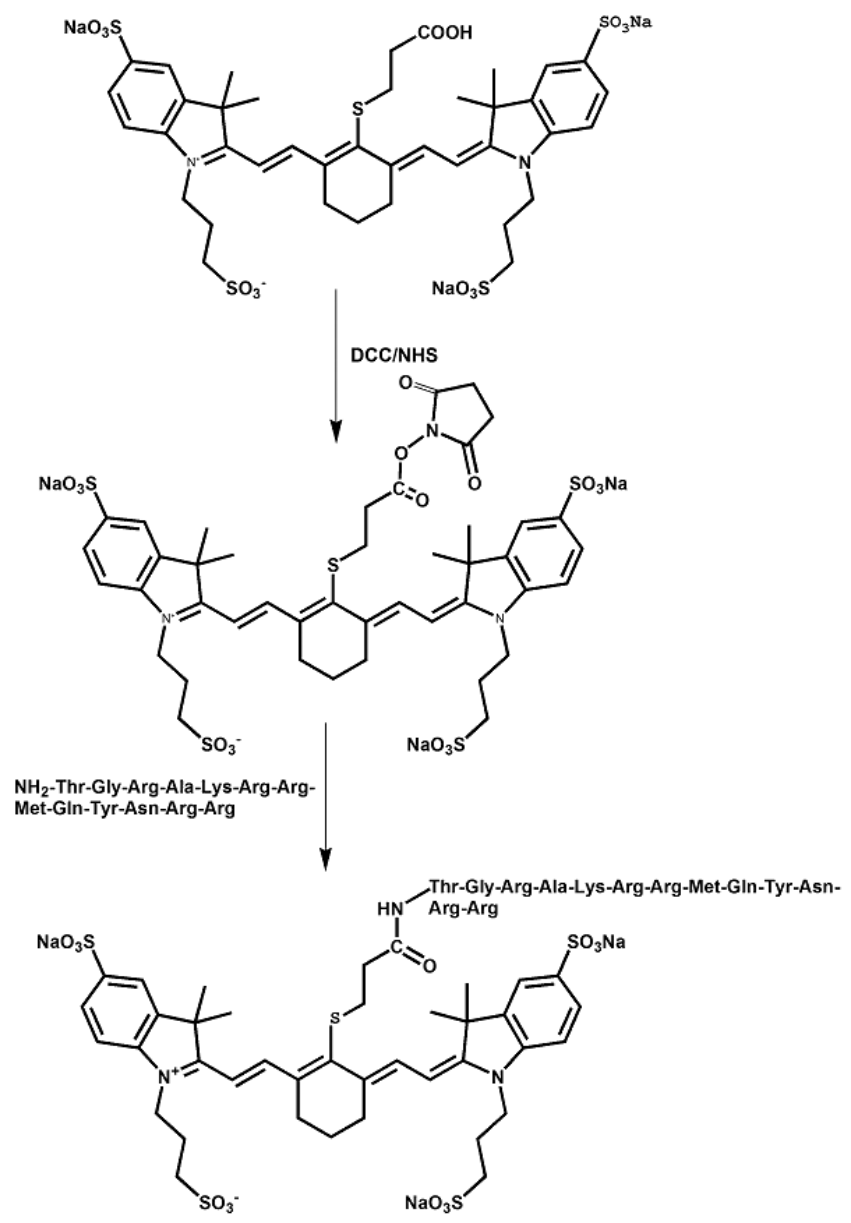

Fig. 1. Synthesis of UBI29-41-ICG02. 


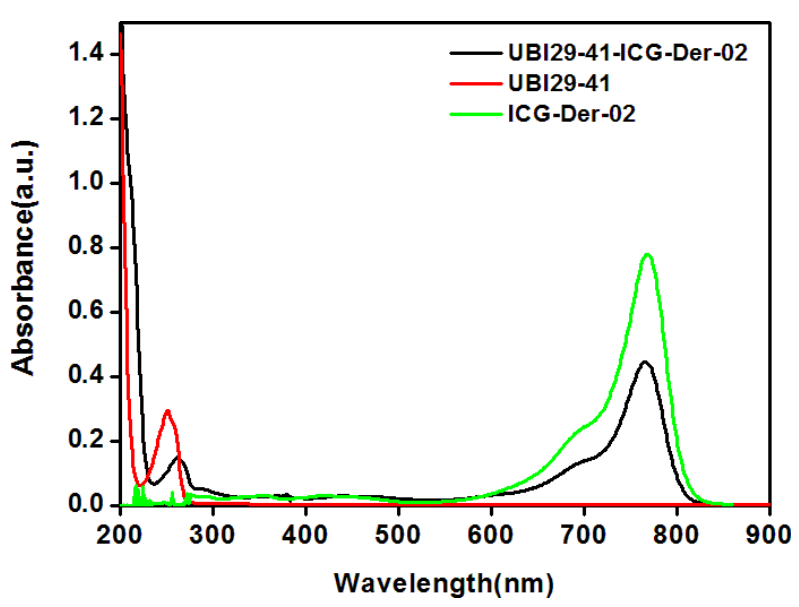

(a)

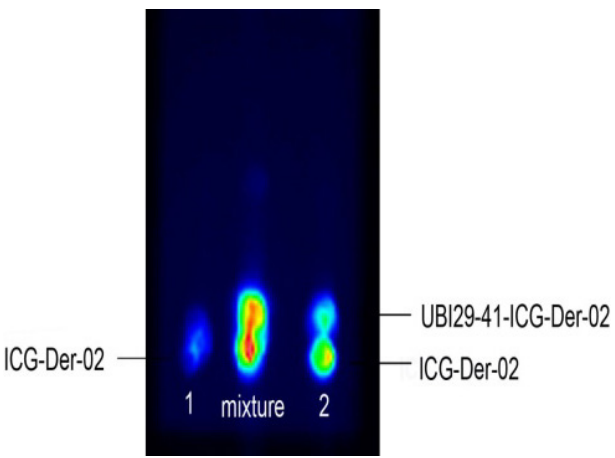

(b)
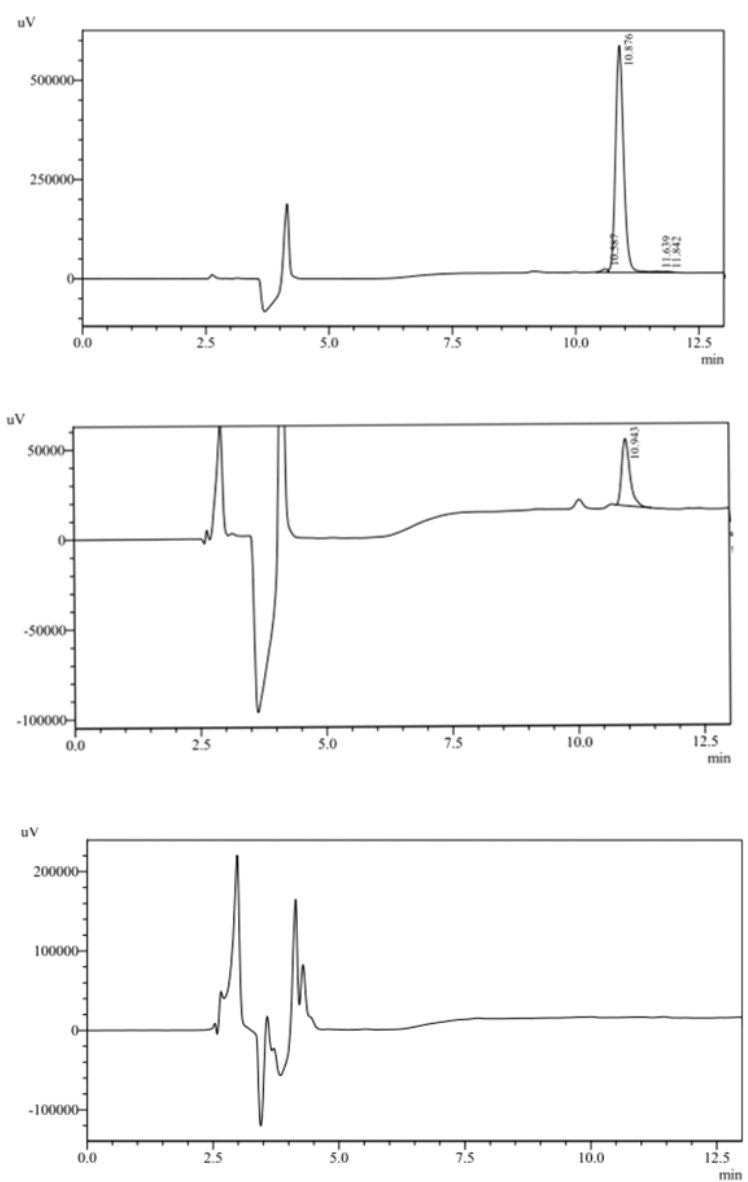

(c)

Fig. 2. Characterization of UBI29-41-ICG02. (a) Absorption spectra of synthesized UBI29-41-ICG02 probe and UBI29-41 and ICG-Der-02 dye. The absorption peaks of UBI29-41-ICG02 overlaps the peaks of UBI29-41 and ICG-Der-02 at 256 and $780 \mathrm{~nm}$, indicating the successful conjugation of UBI29-41-ICG02. (b) TLC (dichloromethane: methanol=5:1) of the UBI29-41-ICG02. The two right spots from the reactants on the right column represents the unreacted ICG-Der-02 (bottom spot) and the product UBI2941-ICG02 (top spot). (c) HPLC profiles of UBI29-41-ICG02. The top is UBI29-41. The middle was the physical mixture of the UBI29-41 and the ICG-Der02. The bottom was the conjugate UBI29-41-ICG02. And the retention time on analytical HPLC for UBI29-41 was found to be $10 \mathrm{~min}$. 
the absorption peaks of the constituent components (UBI29-41 and ICG-Der-02 at 256 and $780 \mathrm{~nm}$, respectively) in the complex, indicating the successful conjugation of UBI29-41-ICG02. The product UBI29-41-ICG02 was characterized by TLC and the fluorescence images were taken by the NIR imaging system, as shown in Fig. 2(b). The two right spots from the reactants on the right column represents the unreacted ICG-Der-02 (bottom spot) and the product UBI29-41-ICG02 (top spot). The product UBI29-41-ICG02 was analyzed by HPLC, as shown in Fig. 2(c). The retention time on analytical HPLC for UBI 29-41 was found to be $10 \mathrm{~min}$. The yield of UBI29-41-ICG02 conjugate was calculated at about $35 \%$ and the purity of UBI29-41-ICG02 was about $90 \%$ from analytical HPLC analysis.

\subsection{In vitro cytotoxicity of UBI29-41-ICG02}

MTT assay was used to quantify the cell viability of the human hepatic L02 cells in the presence of UBI29-41-ICG02 to display its toxicity in vitro. The results are displayed in Fig. 3(a). When the concentration is below $10^{-6} \mathrm{~mol} / \mathrm{L}$, the cell viability was above $80 \%$. When the concentration of the UBI29-41-ICG02 was increased, the toxicity augmented. The MTT assay showed that the probe

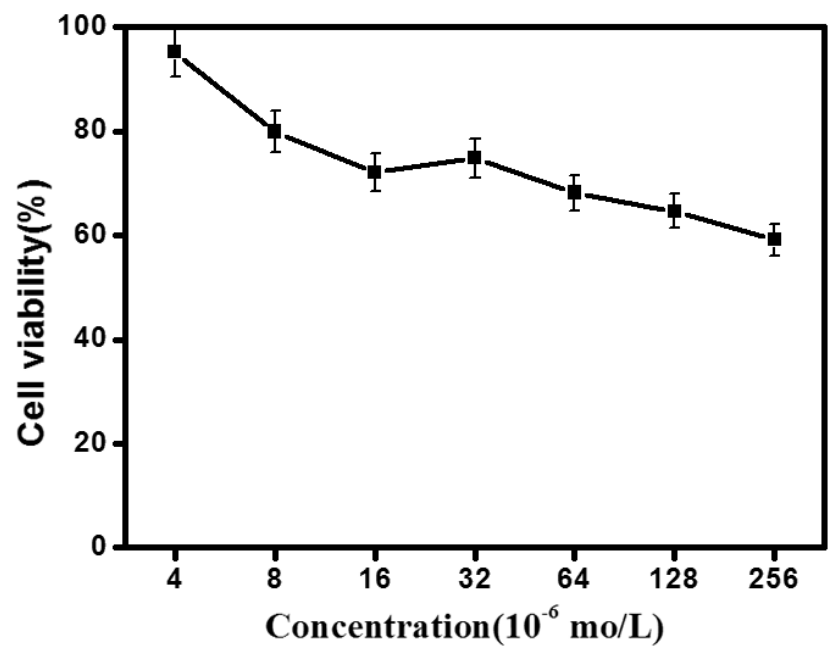

(a) displayed some toxicity to human hepatic L02 cells to some extent. However, because this assay was performed limited times, this result should be replicated again to confirm it again.

\subsection{In vitro antibacterial activity of UBI29-41-ICG02}

We were interested in seeing whether UBI29-41ICG02 still had an antibacterial ability against S. aureus. As shown in Fig. 3(b), there is no obvious inhibition ability to $S$. aureus. Because UBI29-41 is only a fragment of antimicrobial peptide UBI, it did not conserve the antibacterial ability of the ubiqubin. This advantage makes it perfect for imaging bacterial infection in vivo, because imaging signal will decrease if the bacteria are killed.

\subsection{Dynamics and biodistribution of ICG-Der-02 and UBI29-41-ICG02 in healthy mice}

To better understand the physiological behavior of the UBI29-41-ICG02 probe, the dynamics and biodistribution of ICG-Der-02 and UBI29-41-ICG02 were first investigated in normal mice $(n=5)$ prior to using the infection model. These mice were injected with ICG-Der-02 and UBI29-41-ICG02 probe

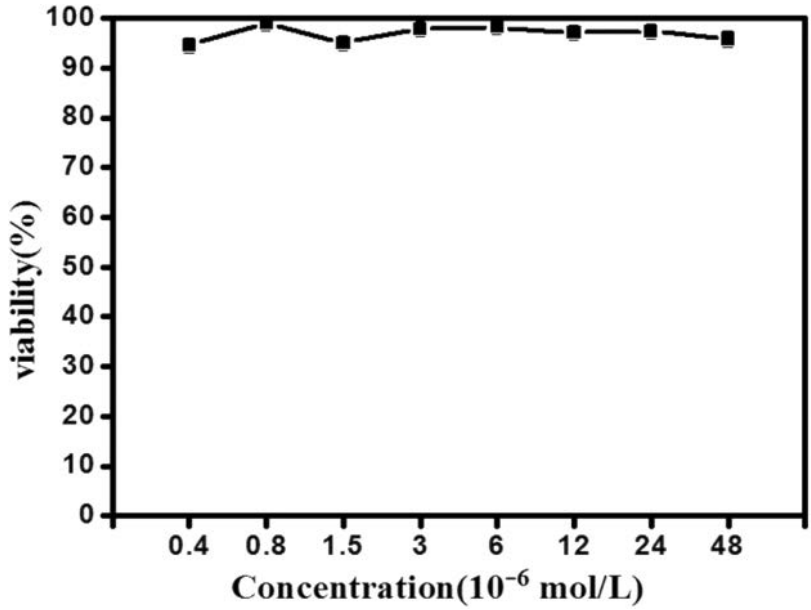

(b)

Fig. 3. The toxicity of UBI29-41-ICG02 to L02 cells and the antibacterial studies to S. aureus. (a) The toxicity to L02 cells. With the concentration of UBI29-41 increased, the toxicity increases too. When the concentration is below $8 \times 10^{-6}$ mol/L, the cell viability is above $80 \%$ and there is no obvious toxicity. (b) The viability of $S$. aureus in different concentration of UBI29-41. From $0.4 \times 10^{-6} \mathrm{~mol} / \mathrm{L}$ to the maximum concentration of $48 \times 10^{-6} \mathrm{~mol} / \mathrm{L}$, there is no inhibition to the growth of the $S$. aureus. 

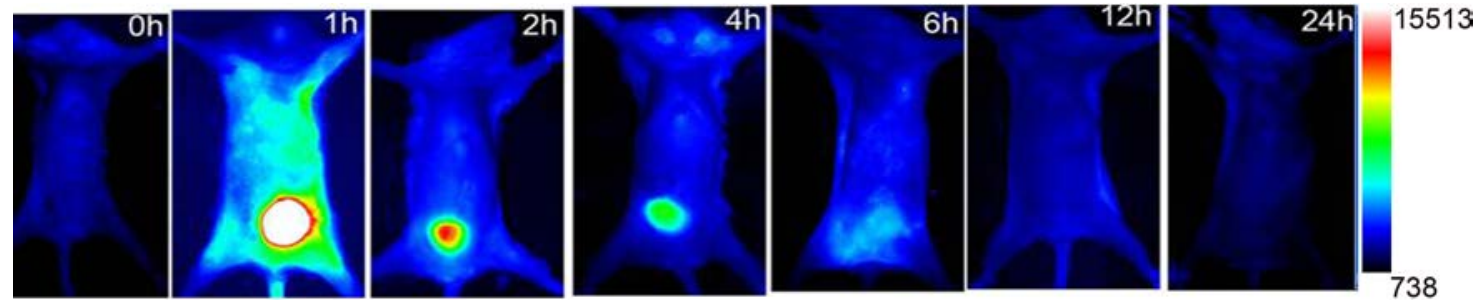

(a)

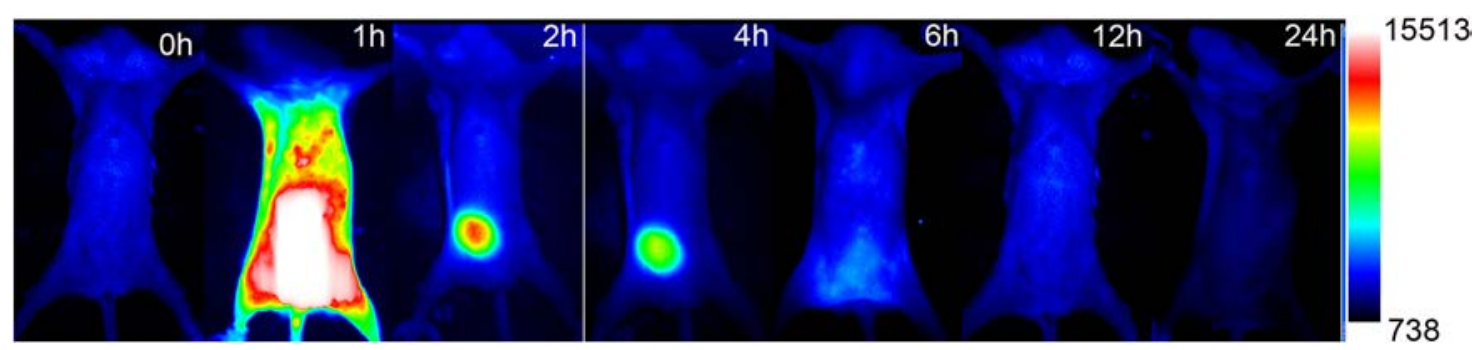

(b)
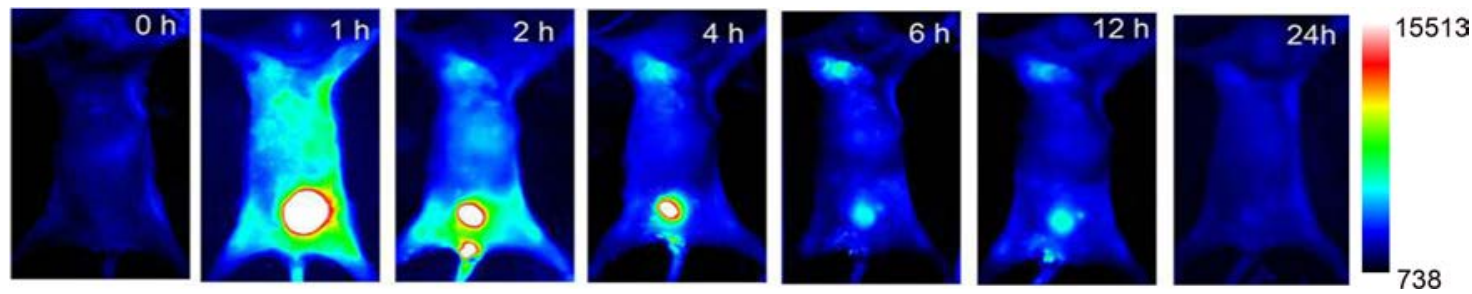

(c)
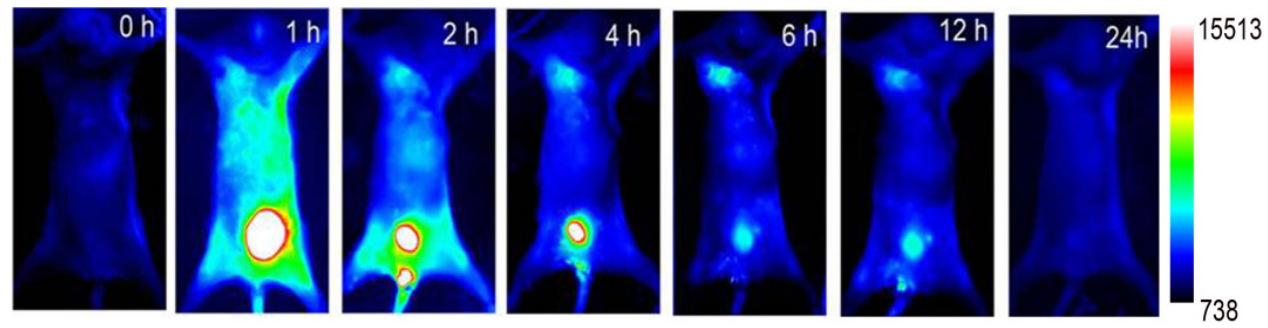

(d)

Fig. 4. NIR images of Balb/C mice. The distribution of the probe UBI29-41-ICG02 (a) and the dye ICG-Der-02 (b) in healthy mice and the distribution of the probe UBI29-41-ICG02 (c) and the dye ICG-Der-02 (d) in infected mice, and the infection site is on the right axillary fossa.

$(0.2 \mathrm{~mL})$, and monitored for $24 \mathrm{~h}$. Representative images are shown in Figs. 4(a) and 4(b).

In vivo imaging shows the fluorescence signals of the probe UBI29-41-ICG02 spread all over the mice at $1 \mathrm{~h}$ and predominantly accumulated in bladder afterward. There was no observable fluorescence signal in the liver. The bright signal in bladder inferred that the probe cleared through the kidneys.

\subsection{Dynamics and biodistribution of ICG-Der-02 and UBI29-41-ICG02 in infected mice}

To investigate the bacteria targeting capability of UBI29-41-ICG02, the $S$. aureus was injected in the right axillary fossa and lower flank, respectively $(n=10)$. The in vivo distribution of the dye ICGDer-02 and probe UBI29-41-ICG02 at specific time 
points was recorded with our NIR imaging system. Representative images are shown in Figs. 4(c) and 4(d). Similar to the profiles of the dynamics study described above, the probes were initially distributed all over the body and subsequently cleared by the renal pathways. However, the infection sites were identifiable within $1 \mathrm{~h}$ postinjection of the probes. Over time, the probe increasingly accumulated in the infection and the fluorescence intensity peaked at about $6 \mathrm{~h}$. The bright fluorescence signal gradually disappeared after $12 \mathrm{~h}$.

\section{Discussion}

In agreement with selective binding of peptides to microorganisms over mammalian cells, the peptide UBI29-41 did not visualize sterile inflammatory processes initiated by a single injection of LPS or heat-killed microorganisms. ${ }^{1,27,28}$ It accumulated significantly higher in the bacterial infection compared to that of the LPS-induced inflammation. It suggests that UBI29-41 based imaging probe may be a promising imaging agent to distinguish between bacterial infections and sterile inflammations. And next we will undertake this research to confirm this possibility.

UBI29-41-ICG02 is composed of two watersoluble components, ICG-Der-02 and UBI29-41. Therefore, UBI29-41-ICG02 targeted to the bacteria faster and quickly cleared from the rodent through the kidney pathway after $6 \mathrm{~h}$.

In the MTT assays, the UBI29-41 shows some toxicity to human hepatic L02 cells. Because UBI2941 is a cationic antimicrobial peptides, the cationic properties may be the factor of the toxicity. ${ }^{29}$ So in the animal experiments, we should control the concentration of the UBI29-41. Besides, we should also determine the toxicity of the probe UBI29-41-ICG02 to other human normal cells next to confirm its toxicity again. The bacteria to detect should not be limited to $S$. aureus, and other common pathogen should also be included in our experiments.

\section{Conclusion}

Overall, this study demonstrates that the NIR fluorescent UBI29-41 probe provides a powerful strategy for noninvasive monitoring of biological dynamics and infection diagnosis in different conditions in vivo.

\section{Acknowledgment}

The authors are grateful to Natural Science Foundation Committee of China (NSFC 81220108012, 81171395, 81071194, 81000666, 30970776, 30672015, 30800257 and 31050110123$)$, the Ministry of Science and Technology (2009ZX09310-004) and the Priority Academic Program Development of Jiangsu Higher Education Institutions for their financial support.

\section{References}

1. A. Lupetti, M. M. Welling, E. K. J. Pauwels et al. "Radiolabelled antimicrobial peptides for infection detection," Lancet Infect. Dis. 3(4), 223-229 (2003).

2. F. D. Lowy, "Antimicrobial resistance: The example of Staphylococcus aureus," J. Clin. Invest. 111(9), 1265-1273 (2003).

3. M. L. Embleton, S. P. Nair, B. D. Cookson et al., "Selective lethal photosensitization of methicillinresistant Staphylococcus aureus using an Ig G-tin (IV) chlorine6 conjugate," J. Antimicrob. Chemother. 50(6), 857-864 (2002).

4. X. H. Ning, S. J. Lee, Z. R. Wang et al., "Maltodextrin-based imaging probes detect bacteria in vivo with high sensitivity and specificity," Nat. Mater. 10, 602-607 (2011).

5. A. Lupetti, P. H. Nibbering, Welling et al., "Radiopharmaceuticals: New antimicrobial agents," Trends Biotechnol. 21, 70-73 (2003).

6. S. J. Jang, Y. J. Lee, S. Lim et al., "Imaging of a localized bacterial infection with endogenous thymidine kinase using radioisotope-labeled nucleosides," Int. J. Med. Microbiol. 302(2), 101-107 (2012).

7. W. M. Leevy, S. T. Gammon, H. Jiang et al., "Optical imaging of bacterial infection in living mice using a fluorescent near-infrared molecular probe," J. Am. Chem. Soc. 128(51), 16476-16477 (2006).

8. M. Zasloff, "Antimicrobial peptides of multicellular organisms," Nature 415(6870), 389-395 (2002).

9. K. Matsuzaki, "Why. How are peptide-lipid interactions utilized for self-defense? Magainins and tachyplesins as archetypes," Biochim. Biophys. Acta. 1462, 1-10 (1999).

10. E. Guaní-Guerra, T. Santos-Mendoza, S. LugoReyes et al., "Antimicrobial peptides: General overview and clinical implications in human health and disease," Clin. Immunol. 135, 1-11 (2010).

11. J. R. Buscombe "The future of infection imaging," Q. J. Nucl. Med. Mol. Imag. 50, 99-103 (2006).

12. G. Ferro-Flores, B. E. Ocampo-Garcia, L. MelendezAlafort, "Development of specific radiopharmaceuticals for infection imaging by targeting infectious 
micro-organisms," Curr. Pharm. Des. 18(8), 10981106 (2012).

13. A. Lupetti, M. M. Welling, E. K. J. Pauwels et al., "Radiolabelled antimicrobial peptides for infection detection," Lancet Infect. Dis. 3(4), 223-229 (2003).

14. M. Gandomkar, R. Najafi, M. Shafiei et al., "Clinical evaluation of antimicrobial peptide $[99 \mathrm{~m}$ Tc/ Tricine/HYNIC] ubiquicidin $29-41$ as a humanspecific infection imaging agent," Nucl. Med. Biol. 36(2), 199-205 (2009).

15. L. Sarda-Mantel, A. Saleh-Mghir, M. M. Welling et al., "Evaluation of 99mTc-UBI 29-41 scintigraphy for specific detection of experimental Staphylococcus aureus prosthetic joint infections," Eur. J. Nucl. Med. Mol. Imaging 34(8), 1302-1309 (2007).

16. P. H. Nibbering, M. M. Welling, A. PaulusmaAnnema et al., "99mTc-Labeled UBI 29-41 peptide for monitoring the efficacy of antibacterial agents in mice infected with Staphylococcus aureus," J. Nucl. Med. 45(2), 321-326 (2004).

17. L. Meléndez-Alafort, A. Nadali, G. Pasut et al., "Detection of sites of infection in mice using $99 \mathrm{~m}$ Tc-labeled $\mathrm{PN}_{2} \mathrm{~S}-\mathrm{PEG}$ conjugated to UBI and $99 \mathrm{~m}$ Tc-UBI: A comparative biodistribution study," Nucl. Med. Biol. 36(1), 57-64 (2009).

18. W. M. Leevy, S. T. Gammon, J. R. Johnson et al., "Noninvasive optical imaging of Staphylococcus aureus bacterial infection in living mice using a bisdipicolylamine-zinc(II) affinity group conjugated to a near-infrared fluorophore," Bioconjug. Chem. 19, 686-692 (2008).

19. T. Hamaoka, K. K. Mccully, "Muscle research work with britton chance from in vivo magnetic resonance spectroscopy to near-infrared spectroscopy," $J$. Innov. Opt. Health Sci. 04(03), 227-237 (2011).

20. B. Zhu, E. V. A. M. Sevick-Muraca, "Minimizing excitation light leakage and maximizing measurement sensitivity for molecular imaging with near-infrared fluorescence," J. Innov. Opt. Health Sci. 4(03), 301-307 (2011).

21. A. C. Merzagora, M. T. Schultheis, B. Onaral et al. "Functional near-infrared spectroscopy-based assessment of attention impairments after traumatic brain injury," J. Innov. Opt. Health Sci. 4(03), 251-260 (2011).

22. W. M. Leevy, N. Serazin, B. D. Smith, "Optical imaging of bacterial infection models," Drug Discov. Today Dis. Models 4(3), 91-97 (2007).

23. J. Cao, S. N. Wan, J. M. Tian et al., "Fast clearing RGD-based near-infrared fluorescent probes for in vivo tumor diagnosis," Contrast Media Mol. Imaging 7(4), 390-402 (2012).

24. A. F. Radovic-Moreno, T. K. Lu, V. A. Puscasu et al., "Surface charge-switching polymeric nanoparticles for bacterial cell wall-targeted delivery of antibiotics," ACS Nano 6(5), 4279-4287 (2012).

25. H. Y. Chen, S. S. Cui, Z. Tu et al., "In vivo monitoring of organ-selective distribution of $\mathrm{CdHgTe} /$ $\mathrm{SiO} 2$ nanoparticles in mouse model," J. Fluoresc. 22(2), 699-706 (2012).

26. L. L. Shan, S. S. Cui, C. L. Du et al., "A paclitaxelconjugated adenovirus vector for targeted drug delivery for tumor therapy," Biomaterials 33(1), 146-162 (2012).

27. M. S. Akhtar, J. Iqbal, M. A. Khan et al., " $99 \mathrm{mTc}$ labeled antimicrobial peptide ubiquicidin (29-41) accumulates less in Escherichia coli infection than in Staphlococcus aureus infection," J. Nucl. Med. 45(5), 849-856 (2004).

28. P. H. Nibbering, M. M. Welling, P. J. Van den Broek et al., "Radiolabelled antimicrobial peptides for imaging of infections: A review," Nucl. Med. Commun. 19(12), 1117-1122 (1998).

29. M. R. Yeaman, N. Y. Yount, "Mechanisms of antimicrobial peptide action and resistance," Pharmacol. Rev. 55(1), 27-55 (2003). 